\title{
Frontières
}

\section{Le legs de la peur}

\section{Wilfrid Noël Raby}

Volume 12, numéro 2, printemps 2000

Peur bleue...

URI : https://id.erudit.org/iderudit/1074394ar

DOI : https://doi.org/10.7202/1074394ar

Aller au sommaire du numéro

Éditeur(s)

Université du Québec à Montréal

ISSN

1180-3479 (imprimé)

1916-0976 (numérique)

Découvrir la revue

\section{Citer cet article}

Raby, W. N. (2000). Le legs de la peur. Frontières, 12(2), 20-27.

https://doi.org/10.7202/1074394ar

\section{Résumé de l'article}

La peur influence nos comportements individuels et collectifs qui en retour propulsent l'histoire humaine. Après un aperçu de quelques indices laissant entrevoir le rôle probable de la peur dans l'émergence de la cité, nous examinerons en détail l'expérience de la peur. Puis nous aborderons son impact sur la mémoire, sur le développement du cerveau de l'enfant. Nous verrons ensuite jusqu'à quel point les structures mentales de la peur sont reproduites dans nos systèmes de droits et de devoirs, pour conclure que la sauvegarde de nos démocraties passe par l'apprivoisement et la remémoration de la peur.
Ce document est protégé par la loi sur le droit d'auteur. L'utilisation des services d'Érudit (y compris la reproduction) est assujettie à sa politique d'utilisation que vous pouvez consulter en ligne.

https://apropos.erudit.org/fr/usagers/politique-dutilisation/ 


\section{Résumé}

La peur influence nos comportements individuels et collectifs qui en retour propulsent l'histoire humaine. Après un aperçu de quelques indices laissant entrevoir le rôle probable de la peur dans l'émergence de la cité, nous examinerons en détail l'expérience de la peur. Puis nous aborderons son impact sur la mémoire, sur le développement du cerveau de l'enfant. Nous verrons ensuite jusqu'à quel point les structures mentales de la peur sont reproduites dans nos systèmes de droits et de devoirs, pour conclure que la sauvegarde de nos démocraties passe par l'apprivoisement et la remémoration de la peur.

Mots clés: peur - circuits neuronaux développement émotif de l'enfant droits de la personne

\section{Abstract \\ Fear motivates individual and collective behaviors, which in turn drive human his- tory. After an overview of a few indica- tors affording us a glimpse into the like- ly role of fear in the appearance of the city, we shall examine the fear experien- ce in detail. Next we will embark upon its impact on memory and the development of children's brains. We will then see up to what point mental structures of fear are reproduced in our systems of rights and duties, concluding that the safe- guard of our democracies goes through taming and recalling fear.}

Key words: fear - neuronal circuits child emotional development - human rights

\section{A R T I C L E}

\section{Le legs de la peur}

Wilfrid Noël Raby,

assistant professeur de psychiatrie et de neurologie, Columbia University, psychiatre titulaire du New York State Psychiatric Institute.

Caïn ayant tué Abel, il chercha refuge contre les condamnations de son Dieu et de sa conscience. Il commença par s'exiler au bout du monde, il se réfugia à la façon des Bédouins derrière des boucliers de peaux, puis de bronze; mais en vain. Torturé, il ordonna à ses fils de lui ériger une ville ceinte de remparts où il trouverait enfin la quiétude. Voici comment Victor Hugo, dans un poème intitulé «La Conscience» évoque ce récit biblique:

Bâtissons une ville avec sa citadelle.

Bâtissons une ville et nous la fermerons.

Alors Tubalcaïn, père des forgerons,

Construisit une ville énorme et surhumaine. [...]

L'ombre des tours faisait la nuit dans les campagnes;

Ils donnèrent aux murs l'épais-

seur des montagnes;

Et sur la porte on grava:

"Défense à Dieu d'entrer»".

Malgré les tours et les murs que sa crainte de Dieu lui avait fait ériger, Caïn ne trouva ni la paix, ni le repos qu'il recherchait. En nous décrivant ainsi le total des oeuvres et des travaux entrepris par Caïn, ses fils, et ses serviteurs, Hugo laisse entrevoir une vérité sur laquelle glissent les récits historiques: à savoir que l'émotion propulse l'histoire parce qu'elle meut la vie consciente et inconsciente de ses acteurs. La peur, émotion primale, bien sise dans ce qu'il y a de plus primitif dans notre cerveau, colore tout ce qu'il y a d'humain. Parce qu'elle concerne la protection de soi, un homme qui ne chérit pas sa vie n'a pas besoin de la peur. Du moment que la vie nous anime, il nous faut la peur pour la garder. Mais il y a plus. Comme la vie humaine s'exprime dans des choses qui transcendent la simple existence, on peut présumer que la peur devrait se manifester dans notre propre oeuvre de création; que ce soit en art, en politique, ou dans toute forme de civilisation; reflétant l'adage que "qui ne craint rien ne s'occupe, ni ne se préoccupe de rien".

Dans cet article, nous tenterons de suivre la trace de la peur à partir de notre biologie qu'elle imbibe, dès le processus perceptif de prise de conscience de ce qui effraie, pour examiner en quoi elle forge notre empreinte sur le monde. Ainsi, après avoir décrit les paramètres biologiques de la peur, l'estampe qu'elle laisse dans notre mémoire, nous montrerons comment les notions contemporaines de droits et de devoirs plongent leurs racines dans l'émotion, notamment la peur; et que son assuétude est pour les sociétés le levain même de leur durée. Nous proposerons aussi que le fait de contenir la peur, d'en saisir le sens, y compris de se la remémorer, est absolument nécessaire à la sauvegarde de nos démocraties. Nous conclurons qu'en définitive, nous ne pouvons pas plus nous passer de la peur, que nous pouvons nous échapper de ce que nous sommes, ni de la biologie qui nous fait.

\section{LA PEUR CONSTRUITE}

L'histoire humaine se distingue entre autres par ses constructions: ses gites, ses village et ses villes. L'agglomération de ces lieux de demeure couva un urbanisme latent, mais nécessaire, car il fallut inventer une façon de se distancier sans cesse de la société nomade. Ainsi l'abandon de la vie nomade semble s'être fait de concert avec la découverte d'un blé primitif, plus luxuriant et productif celui-là, probablement le produit de croisements génétiques fortuits qu'Aeole favorisa entre les herbes diverses qui couvraient les oasis de Jéricho ${ }^{2}$. En plus du concours de la nature, Lewis Mumford dans son oeuvre The City in History ${ }^{3}$ fait aussi remarquer que 
l'agglomération d'un nombre croissant de personnes en un seul lieu n'aurait pu se produire sans la technique:

La nouveauté de l'alliage urbain pava la voie à une immense expression des potentialités humaines et ce, tous azimuts. La cité mobilisa la force de travail humain, aiguillonna la maîtrise des transports [...] et des communications de longue distance et, caractéristique non négligeable, favorisa un formidable développement de la productivité agricole. (Traduction libre de la rédaction)

Dans le creuset que fut la cité de Jéricho, fondée vers l'an 8000 avant J.C., se produisit donc la levée d'un bouillon de culture d'où proliféra toute une palette d'outils et de coutumes comme la culture du grain, le labour et l'animal de trait, la navigation à voile, la métallurgie du cuivre, le calendrier et l'écriture. Il s'ensuivit ce que Mumford considère l'un des premiers rôles de ces villes naissantes: être le réceptacle des multiples fruits de cette effervescence humaine. La bibliothèque pour le manuscrit, ou le silo pour le grain furent des contenants tôt nécessaires, tout aussi importants l'un que l'autre, et sans lesquels la ville n'aurait pu étendre son influence.

Ce changement d'axe de l'activité humaine transforma radicalement nos rapports avec la nature. Des centres urbains devenus trop grands pour être facilement évacués ou approvisionnés en temps de pénurie se sont découverts vulnérables à la menace, qu'un village ou qu'une famille pouvait jusqu'alors facilement escamoter en se déplaçant vers des cieux plus cléments. Cette inertie de la foule urbaine ne changea en rien l'attente qu'avaient les citadins d'une sécurité assurée. Le contraste entre le souhait qu'ils nourrissaient et la réalité qu'ils constataient leur fit craindre que cette sécurité ne soit qu'un leurre, lequel venait en fait voiler une vulnérabilité mortelle face aux désastres naturels, ou encore face à la guerre. Pour survivre, la cité dut donc recourir aux techniques qui l'ont fait naître, essayant de les propulser vers des ouvrages qui assureraient sa protection et sa survie. Ainsi naquit la machine humaine, des milliers d'hommes se mouvant comme un seul sous la direction d'un monarque pour ériger les remparts, creuser les digues, abattre les forêts et ameublir les terrasses. Pourtant, très tôt, la cité dut canaliser les pulsions anxieuses et agressives de ses habitants. Ces mêmes pulsions influeront sur la sélection de ses chefs. Sera roi qui saura apaiser les craintes, qui comprendra intuitivement que pour que la cité survive, une majorité de ses habitants, ou encore une minorité influente de ses habitants, doit non seulement se trouver bien, mais se sentir protégée.

Ce sentiment de sécurité devint un critère par lequel la cité put juger de son état, tant en ses périodes d'accalmie qu'en ses périodes de crise, parce que ce sentiment - mêlé à un certain tonus civique - était capable de galvaniser et de mobiliser ses habitants et ses chefs. Ainsi, dans la Rome antique, en l'an 458 avant J.-C., le consul Cincinnatus, sollicité par le Sénat pour commander les armées romaines contre les attaques des Achéens, quitta le champ qu'il était en train de labourer et suivit les messagers pour redevenir dictateur de Rome. D'après l'histoire et la légende, il détruisit les armées achéennes en seize jours; puis cette victoire assurée, retourna à sa ferme comme simple citoyen.

C'est ainsi que saisi d'une peur subite devant une menace imminente, Cincinnatus aurait contenu sa peur en ce qu'il la jaugea et sut la canaliser vers la lutte contre l'envahisseur achéen. Comme nous le verrons, ce fut là une réaction parmi d'autres possibles. L'émotion en tant qu'appel à l'action nous invite à nous questionnemer sur la façon qu'ont nos déterminismes biologiques d'influencer nos pensées et nos actes, et ultimement notre histoire. En ce qui concerne la peur, nous examinerons d'abord son caractère subit, c'est-àdire l'instantanéité de son expérience.

\section{L'INSTANT DE LA PEUR}

Lorsque la peur fond sur nous, qu'advient-il? Que se passe-t-il en nous lorsque, au sortir d'une courbe, notre trajectoire heurte de front celle d'un camion remorque? Les manifestations de la peur, nous les connaissons: état soudain d'alerte, dilatation des pupilles, sueurs, pâleur, palpitations cardiaques, augmentation du tonus musculaire, réflexes hyperactifs. De plus, nous les reconnaissons chez les animaux d'autres espèces; si bien en fait que la peur est devenue l'archétype de l'émotion comme sujet d'étude. À l'issue de longues et patientes recherches, on a pu tracer la piste neurologique de la peur.

En raison des liens irrémédiables qui soudent la peur à la vie des animaux, il était plausible de supposer qu'une biologie commune - voire des structures cérébrales communes - puisse rendre compte des manifestations de la peur chez des espèces distinctes. Chez les mammifères à tout le moins, cette supposition se vérifierait. L'une de ces structures, le noyau amygdalien, phylogénétiquement très ancien, détient entre autres rôles celui de générer et d'ajuster la réaction émotive à ce qui est perçu par nos sens. C'est notamment par la perception que débute l'émotion; en ce sens, voir permet non seulement de constater mais aussi de s'émouvoir. Au croisement du cerveau qui constate et du cerveau qui s'émeut se situe donc le noyau amygdalien, en une position cruciale. Si cruciale qu'un groupe de chercheurs japonais a observé que

\section{NÉOCORTEX \\ MÉMOIRE EXPLICITE}

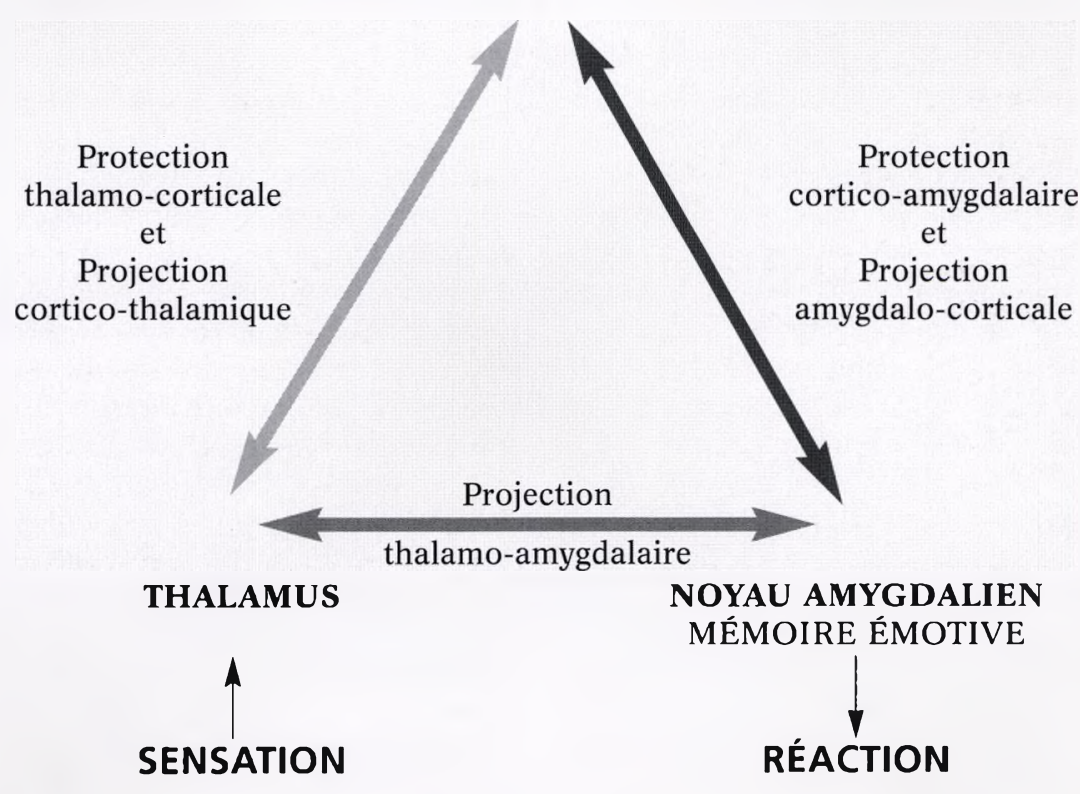


certains habitants de Kolbe, chez qui la maladie d'Alzheimer avait tout particulièrement ravagé le noyau amygdalien, pouvaient se souvenir de certains faits relatifs au tremblement de terre qui rasa leur ville en janvier 1995, mais non pas de la terreur qui frappa leurs concitoyens cette journée-là 4 .

Pour simplifier, le noyau amygdalien reçoit des stimuli sensoriels essentiellement par deux voies: la première par l'entremise de projections neuronales provenant du thalamus; et une seconde, qui conduit les stimuli sensoriels par des projections thalamo-cortico-amygdalaires ${ }^{5}$. Toutes ces connections sont bidirectionnelles, de sorte que le noyau amygdalien, le thalamus et le cortex cérébral sont des structures interreliées ${ }^{6}$.

Chez les mammifères, réagir devant un danger (ce que nous appelons par extrapolation la peur) active simultanément ces deux parcours neuronaux parallèles. Le premier, outrepassant le néocortex, permet une réaction rapide, automatique, réflexe, fondée toutefois sur une information sensorielle approximative. Le second, celui-là relié au néocortex, transmet une information plus détaillée et quantitative, mais qui ne parvient au noyau amygdalien que plusieurs millisecondes après la réception des stimuli issus du thalamus. Alors que le premier parcours permet une réaction rapide, le second parcours cortical évalue la pertinence du réflexe. Il s'ensuit que la perception d'un danger peut activer le noyau amygdalien qui ensuite générera tous les signes physiologiques de la peur, avant même que la notion d'un danger ait atteint la pensée. Réagir à un danger devient possible avant même d'en connaître la teneur.

Au sortir de la courbe donc, l'image du camion remorque éperonne votre cerveau, surtout votre noyau amygdalien. Vous palpitez de peur, mais surtout, vous tournez le volant sans penser. Vous avez bien fait, car vous constatez trois secondes plus tard qu'il s'agissait bien d'un dix-huit roues qui vient de rayer la peinture de votre voiture au passage. Vous avez eu les réflexes efficaces.

Toutefois, à ce trop simple schéma décrivant la réciprocité des circuits neuronaux entre le néocortex, le noyau amygdalien et le thalamus, se superposent des complexités nouvelles, entre autres celle-ci: comme l'arrivée au noyau amygdalien des stimuli thalamiques précède de plusieurs millisecondes l'arrivée des stimuli corticaux, les stimuli thalamiques peuvent modifier et même estomper l'influence des stimuli corticaux sur le noyau amygdalien. Le neurobiologiste Joseph Ledoux ${ }^{7}$ présente ainsi les conséquences possibles:

Il est facile d'imaginer que des différences constitutionnelles et génétiques pourraient conduire à des variations individuelles quant à la dominance soit des projections thalamiques, soit des projections corticales, lorsque se met en branle le traitement de l'émotion par l'intermédiaire du noyau amygdalien. Si ceci s'avère exact, il en résulterait des différences dans le contrôle qu'un individu pourrait exercer sur sa vie émotive. (Traduction libre de l'auteur).

Comme l'anatomie "émotive» du cerveau le suggère, le caractère subit de la peur déclenche notre comportement sans que nous en ayons pleinement conscience. C'est pourquoi il devient vite impossible de discuter davantage de la peur sans aborder les thèmes de la conscience et de l'inconscient, et des mémoires distinctes qui y résident à demeure.

\section{MÉMOIRE, CONSCIENCE ET INCONSCIENT}

Freud énonça deux idées capitales en ce qui concerne les liens entre l'inconscient et la conscience. Il proposa premièrement que nous n'avons pas conscience d'un vaste panorama de notre vie mentale, qu'il s'agisse soit de nos perceptions, de nos rêves ou de nos désirs. Deuxièmement, il soutint que notre inconscient noue chaque événement psychique au précédent, créant ainsi le canevas sans cesse plus serré de nos vies psychiques ${ }^{8}$. Il sous-entendait ce faisant que rien en notre vie mentale n'est laissé au hasard et qu'il existe non seulement une mémoire consciente ou explicite, mais aussi une mémoire inconsciente, incluant une mémoire dite implicite.

Nous devons à Brenda Milner de l'Institut Neurologique de Montréal la découverte des assises de la mémoire consciente et l'élaboration des concepts de mémoire implicite et explicite ${ }^{9}$. Par mémoire explicite, elle désigne la mémoire consciente des êtres, des lieux, des événements et des choses. Celle-ci dépend largement du fonctionnement intégral de l'hippocampe. La mémoire implicite, quant à elle, regroupe diverses fonctions mnémoniques. En plus de la mémoire émotive il faut $\mathrm{y}$ inclure les réflexes, les conditionnements, les dextérités acquises (comme les techniques) et les habitudes acquises, qui sont tous des aspects de notre comportement pouvant se passer à notre insu. Chacune de ces fonctions fait entrer en jeu des parties différentes du système nerveux: pour les réflexes, la moelle épinière; pour les conditionnements, le noyau amygdalien et le cervelet; pour les dextérités, le diencéphale et les noyaux gris centraux et pour les habitudes, le cortex préfrontal $^{10}$. Toutefois, lorsque les circonstances le requièrent, les deux mémoires fonctionnent de concert. Apprendre à nager demande tout d'abord un effort conscient, qui est graduellement relégué à la mémoire implicite du cervelet à mesure que notre habilité s'accroît. Des interactions de ce genre entre les deux systèmes mnémoniques représentent la norme plutôt que l'exception.

Une autre approche consisterait à associer la peur à ses circonstances et à son contexte. Mémorisée par association, la peur deviendrait anticipable, reconnaissable, et possiblement contournable. Freud $^{11}$ proposa en 1926 que l'appariement de perceptions neutres à des perceptions menaçantes pouvait conduire à ce que des situations exemptes de danger soient perçues comme dangereuses.

L'individu aura considérablement développé sa capacité d'autopréservation s'il peut prévoir et anticiper une situation traumatique qui provoquerait l'impuissance en lui plutôt que de simplement attendre son occurrence.[...] C'est dans cette situation que le signal de l'anxiété est déclenché. (Traduction libre de la rédaction)

Ce fut Ivan Petrovich Pavlov qui appliqua la méthode expérimentale aux mécanismes jusqu'alors inconnus de l'apprentissage par association ${ }^{12}$. En étudiant le réflexe salivaire des chiens, Pavlov démontra que des animaux pouvaient apprendre qu'un timbre sonore précède la présentation de nourriture, au point de saliver à la seule écoute dudit timbre. Le point clé pour Pavlov fut le suivant: le timbre qui annonce la présentation de nourriture est mémorisé comme étant une prédiction ${ }^{13}$. De plus, Pavlov reconnut rapidement que les stimuli aversifs créaient des conditionnements tout aussi persistants, sinon plus. Des rats ayant appris à associer une lumière à l'arrivée d'un choc électrique au plancher de leur cage paniquèrent encore à sa vue, même après un sursis de plusieurs mois pendant lesquels la cage de ces mêmes rats fut dotée de tous les conforts afin de les apaiser ${ }^{14}$.

Étant donné la ténacité du conditionnement, Pavlov et beaucoup d'autres après lui se sont questionnés sur son rôle évolutif. Il apparaît important pour un animal de distinguer consciemment et inconsciemment les événements et les contextes qui favorisent leur existence de ceux qui la menacent. En traitant par 
conditionnement associatif les signes flottant au sein de leur environnement, les animaux parviendraient à décoder leur milieu et à augmenter leur capacité d'y survivre. Eux aussi, comme les humains sans doute, consacreraient une grande partie de leur vie cérébrale à se sécuriser, et peut-être à se sentir mieux dans leur niche écologique.

\section{L'ENFANT}

Il n'y a personne pour qui ces considérations soient plus importantes que pour l'enfant. Tout d'abord, il faut comprendre que les mécanismes mnémoniques de l'enfant sont tous inconscients, et ce jusqu'à l'âge de quatre ou cinq ans. Avant cet âge, les structures cérébrales de la mémoire explicite comme l'hippocampe ne sont pas encore suffisamment formées. Il n'en demeure pas moins que ces premières années jouent un rôle fondamental

L'enfant apprend de ses parents quel impact il cause sur son monde extérieur, et ceci le sensibilise à une réalité bien précise: il existe des personnes autres que lui qui réagissent à sa présence. Une interaction sécurisante crée le ferment de la confiance face au monde extérieur; au contraire, une interaction frigide, voire violente, suscite une anxiété qui perturbera non seulement les relations de l'enfant avec les êtres et les choses, mais la croissance même de son cerveau.

À la fin de la Seconde guerre mondiale, René Spitz compara deux groupes d'enfants séparés de leur mère. Le premier groupe se trouvait dans un orphelinat où chaque infirmière veillait aux soins de sept enfants; une prison pour femme abritait le second et chaque jour, chaque nourrisson recevait les soins de sa mère pendant plusieurs heures. Spitz constata après un an que le développement moteur et intellectuel des enfants de la prison surpassait celui des enfants de l'orphelinat ${ }^{15}$, ce qui l'amena à se questionner sur les différences anatomiques cérébrales que ces résultats laissaient entrevoir.

Le mérite d'avoir systématisé l'ensemble des observations sur le développement de l'enfant revient toutefois à John Bowlby, un psychanalyste anglais. Il élabora le concept d'un système d'attachement par lequel l'enfant emploierait des signaux comportementaux pour soit restaurer, maintenir, ou augmenter son attachement à ses parents. Ce concept est si important pour toute discussion sur la peur qu'il convient de citer Bowlby généreusement ${ }^{16}$.

Le concept de système de comportement régule rend compte de la manière dont un enfant maintient sa relation à sa figure d'attachement entre certaines limites d'éloignement ou d'accessibilité. [...] Il s'agit donc d'une forme d'homéostasie comportementale plutôt que physiologique, par laquelle une progéniture maintient sa relation vitale avec son objet d'attachement, en déterminant ces dites limites de proximité et d'accessibilité. Ce système comportemental, conçu comme permanent, et certainement comme un aspect primordial de la personnalité de l'enfant, n'est jamais au repos. Ce système, pour être efficace, doit être doté de senseurs qui le tiennent constamment informé des événements pertinents qu'il doit suivre de près et évaluer. Ces événements à considérer sont de deux ordres: ceux qui indiquent la présence (interne ou externe) d'un danger ou d'un stress potentiel, et ceux qui ont trait aux sources de confort et de sécurité et à leur accessibilité. [...] Au cours du processus de décision conduisant à une manière d'agir plutôt qu'une autre, il est supposé par ce modèle que le système a recours au schéma de comportement de sa figure d'attachement, déjà engrangé et accessible d'emblée. C'est en postulant l'existence de ces "données cognitives» et leur usage par le système d'attachement que la théorie peut tenter d'expliquer comment les expériences de l'enfant avec ses figures d'attachement en arrivent à influencer son mode d'attachement aux autres. (Traduction libre de la rédaction)

Par l'entremise de son système d'attachement, l'enfant puise parmi les ressources dont disposent les adultes afin d'augmenter ses propres chances de survie. Ce faisant, il se donne une lentille mentale au travers de laquelle il jaugera le monde. Selon ce qu'il puise chez ses parents pour s'abreuver, l'enfant se sentira sécurisé ou effrayé, survivra ou non, et c'est en cela que le parent transmet l'eau qu'il a lui-même puisée, lorsqu'il était enfant.

La peur et l'anxiété transforment radicalement le comportement de l'enfant, comme chaque parent le sait trop bien. Selon Bowlby, lorsque l'enfant est séparé de sa mère, il réagit d'abord en protestant, puis en cédant à une réaction dite "de désespoir». Durant la première phase, l'enfant crie, cherche à s'agripper, pleure, aura une pression sanguine et un pouls élevé, déployant ainsi tous les efforts à sa portée pour se rapprocher. Si la séparation continue, l'enfant en vient à constater qu'on ne lui répond pas; l'anxiété fait place à l'apathie, à ce que Bowlby a qualifié de désespoir. L'activité visant le rapprochement cesse et laisse place à l'inertie d'une survie passive qui conservera l'énergie vitale au cas où la séparation se prolongerait. Le silence, présume-t-on, gardera au loin les prédateurs soudainement de passage.

Il semble qu'un système d'attachement comme le postule Bowlby est si crucial pour l'enfant et pour la succession des générations qu'on en découvre des facettes chez d'autres espèces, entre autres chez les rongeurs, où son étude a largement accru notre compréhension. Quand Hans Selye proposa son concept de stress $^{\pi}$, il expliqua que les stresseurs provoquent la sécrétion d'hormones glucocorticoïdes par les glandes surrénales et que c'est cette sécrétion qui permet au corps humain de tolérer le stresseur, en faisant pencher le métabolisme vers un état catabolique. À des degrés divers, ceci protège le corps contre les effets délétères du stress; s'ils se prolongent toutefois, ces mêmes changements métaboliques usent et corrodent le corps comme un acide. Lorsque, pendant les deux premières semaines de leur vie, pour un laps de temps aussi court que dix minutes par jour, des souriceaux sont séparés de leur mère, ils émettent aussitôt des cris qui alertent cette dernière, qui tout de suite se met à leur recherche. Les souriceaux ne cessent pas de crier dès lors que leur mère les trouve; ils continuent au contraire, semblant stimuler la mère à les lécher, à les prendre plus souvent qu'à leur tour, à les replacer dans le nid. Cette intense activité de la mère modifie grandement la réaction des souriceaux au stress de la séparation; particulièrement, elle diminue l'ampleur de la sécrétion de glucocorticoïdes, non pas momentanément ou pour quelques mois, mais pour toute la vie des souriceaux. En d'autres mots, l'activité de la mère transforme la réaction endocrinienne de la peur chez ces muridés pour le reste de leur vie. Une fois adulte, ils se sont montrés moins anxieux et vulnérables face à toute une panoplie de stresseurs. Inversement, si des souriceaux sont soumis au même traitement sans l'intervention de la mère, il y a tout aussi irrémédiablement une augmentation de la sécrétion des surrénales, créant des souris anxieuses et apeurées ${ }^{18}$.

Les répercussions sur le cerveau du système d'attachement ne s'arrêtent pas là. Le cerveau, les neurones de l'hippocampe notamment, exposent à leur surface des récepteurs à glucocorticoïdes qui traduisent la présence de l'hormone dans le sang en effets génétiques et biochimiques au sein de la cellule. Or un stress continu pendant plusieurs 
semaines, jumelé à une sécrétion accrue de glucocorticoïdes, produit une «atrophie» des neurones de l'hippocampe, qui est réversible du moment que le stress s'estompe. Si le stress est maintenu pendant des mois - voire des années - l'atrophie des neurones de l'hippocampe devient irréversible et il s'ensuit une apoptose graduelle de ces neurones, émondant les synapses et les neurones indispensables pour le fonctionnement de la mémoire explicite ${ }^{19}$.

Dans mes propos sur la peur instantanée, j'ai indiqué que l'activité du noyau amygdalien, essentielle aux manifestations de la peur, pouvait modifier l'influence que le néocortex a sur elle, de telle sorte que le contexte et la teneur d'une peur ressentie soient voilés. Nous voici maintenant devant une autre complexité neurologique liée à la peur: l'incarnation de la peur, sa traduction en suractivité endocrinienne, peut compromettre l'activité et le développement de l'hippocampe, de sorte que dès l'enfance l'émergence de la mémoire explicite sera perturbée. La peur inconsciente de l'enfance est donc capable de distiller un poison qui amoindrira les capacités mnémoniques dont nous dépendrons plus tard pour dominer la peur, une fois devenus adultes.

La peur, comme philtre à la fois tonique et poison, est ainsi capable de nous armer ou de nous désarmer devant les vicissitudes de la vie. Quand Cincinnatus, un soldat chevronné, réagit comme il le fit devant la menace, ce fut selon des schèmes conscients et inconscients qui révélaient une confiance de marbre en son identité. Inversement, pour compenser une insécurité profonde sise en une expérience d'abandon et de peur dès l'enfance, il est plausible qu'un individu puisse rechercher chez les autres, individuellement ou au sein d'un groupe, les attributs qui lui semblent faire défaut. Ce comportement s'explique par une particularité du système d'attachement de l'enfant: l'usage des structures mentales et émotives d'un autre, d'un chef, ou d'un groupe pour construire les siennes propres. On trouve ici le germe de la mentalité de groupe. Chez l'adulte souhaitant l'adhésion au groupe, il semble se produire une régression émotive, quasi-inconsciente, à un stade de développement émotif plus caractéristique de celui d'un enfant que d'un adulte capable de discernement. L'impact est parfois favorable: au sein d'un groupe altruiste ou progressiste, l'adhésion peut semer chez l'individu un désir de s'éduquer, un sentiment de fierté et de dévouement dont il serait autre- ment incapable. Cependant, plus la régression tend aux extrêmes, plus le cristal psychologique se fait mince, tout spécialement à la marge du bien et du mal. Lorsqu'il se rompt peut se rompre avec lui toute moralité. Dans son livre The Unconscious Motives of War, Alix Strachey ${ }^{20}$ décrit ce qui peut advenir d'un individu chez qui la peur conduit à une régression psychologique extrême.

L'attachement d'un membre d'un groupe aux autres membres de ce même groupe s'édifie pour une large part en dissolvant tout attachement envers ceux qui ne sont pas membres du groupe. Le membre devient non seulement indifférent au bien-être des non-membres, mais bien plus, il peut en venir à souhaiter leur destruction et leur malheur. [...] Les liens de dépendance et d'identification que le membre forme avec les autres membres de son groupe se créent aux dépens de l'intensité de liens similaires avec des personnes qui ne sont pas membres du groupe. S'il n'en résulte pas une hausse de l'hostilité du membre envers ceux qui ne font pas partie du groupe, à tout le moins peut-il devenir plus indifférent, au détriment des efforts qu'il déploiera pour contenir son hostilité. (Traduction libre de l'auteur)

La peur nous conduit donc de plainpied à la façon dont coexistent le bien et le mal en notre monde. Dans la section suivante, nous verrons comment, à partir de la distinction entre peur et terreur, l'édifice mental de nos peurs pèse sur nos notions de droits et devoirs. Nous verrons de plus que la sauvegarde de nos démocraties passe par le rappel à la conscience de la peur et que, à cet égard, l'art et la littérature deviennent des toniques vitaux pour nous prémunir des déviations et des manipulations de la peur, voire même pour nous protéger contre les assauts de la terreur organisée.

\section{LA PEUR SOCIALE}

Quand, au fil des siècles, la cité assuma une place irrévocable dans la vie quotidienne des hommes et la vie historique des peuples, elle institua un schis me entre ses habitants, lié à la répartition de la richesse. Progressivement, la pratique aidant, puis l'arbitraire décrétant, les biens de la cité ne firent plus l'objet d'une distribution égalitaire. Ils allaient devenir une propriété qui, appartenant aux nantis, pouvait être déniée ou rationnée aux pauvres. Cette notion de propriété, au sens que la cité lui accordait désormais, n'existait pas sous une forme aussi radicale dans la société rurale qui précéda l'apparition des villes. Auparavant, les hommes appartenaient à la terre et partageaient le plus souvent ses fruits dans l'abondance comme dans la pénurie. C'est avec l'érection des cités qu'allaient se produire les premières famines artificielles par lesquelles le riche assurait sa domination sur le pauvre en tenant bien en main - avec le concours des armées les rênes de l'approvisionnement en victuailles. La propriété comme principe organisateur de la cité apparut avec le couronnement des rois, si l'on en croit Lewis Mumford ${ }^{21}$.

La notion de propriété privée requiert que la propriété commune soit considérée comme le bien privé du roi, dont la vie et la prospérité s'identifiaient à celles de la cité. La propriété devenait en quelque sorte un symbole magnifié de la personnalité royale. [...] Une fois cette proposition acceptée, la propriété pouvait donc être retirée du bien commun et octroyée comme récompense du roi pour services rendus. Une fois la propriété retirée du bien commun, elle pouvait être léguée, subdivisée ou vendue pour obtenir un profit privé. [...] Au sein de la cité, le droit de propriété reçut une sanction spéciale, [...] souvent plus sacrée que la vie même. Afin de protéger le droit de propriété, les rois n'hésitèrent pas à recourir à la torture. [...] Ceci représentait, comme la guerre, un degré nouveau de férocité propre à la culture urbaine. (Traduction libre de l'auteur)

La cité fut donc fondée sur une culture de violence, inconnue jusqu'alors. Le climat de suspicion qui en résulta ainsi que les rébellions suivies de violentes répressions qui éclatèrent à l'occasion - comme les soulèvements des plèbes romaines à la fin des tribunats cléments de Tibérius Gracchus (133 avant J.-C.) et de Caius Gracchus (121 avant J.-C. $)^{22}$ - allaient justifier la mise en place de tout un code juridique et philosophique autour de la notion de propriété et pour sa défense.

Ce furent les philosophes empiristes du XVIIe siècle qui établirent le contrôle de la nature humaine comme prérequis aux institutions politiques, y compris la propriété. John Locke fut le plus influent de ce groupe de philosophes, et aussi le plus confiant en la capacité des hommes à coexister pacifiquement. Nonobstant cette prémisse, il n'appréhendait pas moins la menace d'un individualisme sans bornes.

La liberté des hommes gouvernés consiste à disposer de règles balisant la vie quotidienne, applicables à tous, et décrétées par le pouvoir législatif; restreignant la liberté individuelle d'agir 
selon son bon plaisir; ces règles protègent chacun contre l'inconstance et l'arbitraire de la volonté des autres ${ }^{23}$. (Traduction libre de l'auteur)

Thomas Hobbes, pour sa part, entretenait une vision plus pessimiste, certains ont dit plus lucide, de la nature humaine. Il décrivait les hommes comme foncièrement égoïstes; seul un pouvoir absolu, entériné par un contrat social, pouvait à la fois les protéger contre eux-mêmes, et satisfaire une partie de leurs appétits égoïstes ${ }^{24}$.

La condition de l'homme est celle d'une guerre perpétuelle contre son semblable, dans lequel cas chacun est gouverné par sa propre raison. [...] Il s'ensuit de cette condition que chaque homme a le droit à chaque chose, même au corps d'autrui. Par conséquent, aussi longtemps que le droit naturel de chaque homme d'avoir toute chose continue, il ne peut exister aucune sécurité pour quiconque, [...] ni fruit pour quelconque labeur, ni propriété. (Traduction libre de l'auteur).

Hobbes craignait que sans un gouvernement absolu, le climat latent de violence qui entoura la création de la cité ne soit pas contenu, ouvrant ainsi la porte à un régime de terreur sans balises. Certains exemples contemporains de la dissolution soudaine de gouvernements autocratiques semblent lui donner en partie raison. Des Balkans jusqu'en Russie, la fin du communisme a permis l'expression de tous les degrés de violence, de l'anarchie, du crime sordide jusqu'à la terreur pure. Depuis, devant la perte de leurs attaches à un gouvernement omniprésent, les Russes semblent aux prises avec une réaction «bowlbyienne" de désespoir. En dix ans, la Russie a dû faire face à la fois à une chute de l'espérance de vie chez les aînés et à une hausse du taux de suicide chez les jeunes ${ }^{25}$. Livrés à une terreur anarchique suite à l'abandon politique, les Russes tenaillés de peur crient par leurs morts leur désespoir d'être à la dérive, et révèlent ce faisant les points d'ébullition d'une biologie qui ne se laisse pas oublier.

Une distinction s'impose entre la peur et la terreur. La peur étant un état d'être, elle nous tient par les rênes: notre survie en dépend. La terreur est produite par un acte, provoqué ou subi, devant lequel nous nous sentons englués d'impuissance, mais qui pourtant exige que nous réagissions, sans quoi notre cheminement vers la civilisation s'en trouvera compromis. On peut légiférer ou s'armer contre la terreur, on ne peut pas le faire contre la peur. À n'en pas douter toute- fois, la peur influe sur nos actes et donne substance à nos réactions qui soutiennent ou contrecarrent la terreur. L'examen de nos notions modernes de droits et devoirs est éclairant à cet égard.

Considérons deux époques contrastées, le Moyen Âge et le $\mathrm{XX}^{\mathrm{e}}$ siècle. Au temps du régime féodal, les serfs devaient se soumettre au dit "droit de mainmorte», selon lequel ils ne pouvaient disposer de leurs biens par testament.

Quant à son nom, il venait de l'usage odieux de couper la main droite d'un serf décédé pour la présenter au seigneur, qui, de ce moment, s'emparait de tous ses effets, au préjudice et à l'exclusion des enfants de l'homme mort. Puis, comme il fallait faire quelque chose de cette main, les seigneurs les clouèrent à la porte de leurs donjons, à côté de la tête des animaux sauvages tués à la chasse 26 .

En 1949, la «Déclaration universelle des droits de l'homme», s'inspirant en partie de la «Déclaration des droits de l'homme et du citoyen" de la Révolution française, est adoptée par les Nations Unies. On y lit dans le préambule:

Considérant que la méconnaissance et le mépris des droits de l'homme ont conduit à des actes de barbarie qui révoltent la conscience de l'humanité et que l'avènement d'un monde où les êtres humains seront libres de parler et de croire, libérés de la terreur et de la misère, a été proclamé comme la plus haute aspiration de l'homme 27 .

Puis à l'article 5:

Nul se sera soumis à la torture, ni à des peines ou traitements cruels, inhumains ou dégradants. Comment expliquer qu'un tel contraste - comme le droit à l'intégrité corporelle - ait surgi en sept siècles d'histoire? Tout particulièrement, le fait qu'en Occident aujourd'hui, la notion de droits universaux, même lorsqu'elle est ignorée, s'impose désormais comme pilier central du discours politique. Cette notion de droit ne pouvait naître tant que l'individu au sein de sa collectivité n'était pas en mesure de participer de quelque façon que ce soit à l'acte de gouverner. Cette participation, le serf ne l'imagina certes pas, lui dont la vie dépendait entièrement du seigneur, qui la considérait comme une propriété livrée à son bon plaisir. La notion de droit dut attendre les ruptures historiques issues de la Renaissance, dont la
Révolution française; les rênes du pouvoir politique se retrouvant progressivement aux mains d'un plus grand nombre.

Quant à la Déclaration universelle des droits de l'homme, elle fut le cri d'alarme que poussa la cité moderne devant les horreurs de l'Holocauste juif. Sa rédaction appartint à la mémoire explicite de l'horreur et son adoption correspondit à l'émotion du moment comme l'eau à la soif. La notion de droit que cette Déclaration a instaurée dans notre modernité est passée au fil des ans dans la pénombre de la mémoire implicite, en ce qu'elle devint une prémisse sous-entendue, mais attendue de la politique de l'après-guerre. Cette notion de droit s'est muée en un puissant principe oeuvrant dans l'a priori de notre mémoire collective, et qui se manifeste avec constance à tous les niveaux de notre système politique.

La Déclaration universelle des droits mentionne aussi la prérogative des devoirs de l'individu. Article 29 de la Déclaration universelle:

L'individu a des devoirs envers la

communauté dans laquelle seul

le libre et plein développement

de sa personnalité est possible.

Il n'y a pas de droits sans peur surmontée, pas d'avenir pour nos droits sans devoir de les affirmer et de les défendre; et pas de devoirs sans volonté ferme de ne plus être soumis à la tutelle de la terreur organisée. Les outils militaires, diplomatiques et légaux qui protègent nos droits contre les assauts de la terreur ne sont autres choses que les bras agissants de notre mémoire collective, explicite et implicite. Si nous ignorons le sens profond de la peur, nous ignorerons les assises humaines de la terreur, tout comme les assises de nos systèmes de droits et devoirs. Plus encore, nous perdrons la cohésion, si infime soit-elle qui nous permet de vivre en société, puisque nous ne saurons plus comment nous apaiser, nous réconforter, nous solidariser.

\section{MÉMOIRE ET IMAGINAIRE}

Nos notions de droits que recèle notre mémoire collective implicite sont toujours à l'oeuvre, chaque fois plus sûres, plus achevées, avec chaque génération qui considère les droits universels comme allant de soi; comme aussi ordinaires que le sol sous nos pieds. À moins qu'une catastrophe naturelle n'anéantisse les montagnes immobilières de nos villes... Nous tendons à considérer nos créations comme aussi naturelles que le feu, l'eau et l'air, tant elles font partie de notre quotidien. Cette illusion nous sert bien. Il y a 
dans la continuité des démocraties une démarche bien lamarckienne. Dans sa Philosophie Zoologique, Jean Baptiste Monet, chevalier de Lamarck, naturaliste français, explicita sa théorie de l'évolution. Sa théorie des caractères acquis soutenait qu'un organisme acquiert une caractéristique nouvelle en raison de pressions sélectives exercées par l'environnement et que ces caractéristiques peuvent être transmises à la progéniture. Comme biologiste, il vit sa théorie démentie par les travaux de Mendel et de Darwin; mais s'il avait pu être sociologue, on l'aurait couronné pour avoir donné préséance à la transmission des caractères acquis d'une société

Les systèmes de droits et les recours légaux, militaires, politiques et diplomatiques qu'ils sous-tendent, une fois créés et consolidés par une génération, sont transmis in toto à la génération suivante, qui à son tour peut les adapter aux pressions nouvelles qu'elle affronte. Ainsi sont transmis les ajouts et les amendements "acquis» à ces systèmes, un peu comme devait être transmis l'acquis d'un cou allongé d'une génération de girafe à une autre. Le legs de la peur pour une démocratie mûrissante se consolide au fur et à mesure que se succèdent les générations pour qui les droits universels sont affirmés et protégés. À chaque génération, nos démocraties acquièrent en substance et en pesanteur un aplomb sans cesse accru pour affronter la terreur. Cette transmission des "droits acquis» infuse à la démocratie un potentiel de durée et lui confère l'illusion d'appartenir à l'ordre naturel des choses.

Comme nous vivons en aval de la Déclaration universelle des droits, il nous est facile de nous illusionner sur la solidarité apparente de nos droits et sur la vigilance protectrice de nos devoirs. Des drames comme la guerre des Balkans nous rappellent combien ce leurre nous est facile, surtout quand il nous tend le piège de l'oubli. S'il n'y a pas de droits sans peur surmontée, il n'y a pas de survie pour nos droits sans la peur remémorée, sans un outrage qui nous fouette à l'exercice de nos devoirs. On croirait que toute la panoplie des menaces réelles dont nous sommes la cible devrait suffire pour nous garder en alerte. Mais, de la guerre du Vietnam jusqu'au massacre du Rwanda, nous avons l'outrage de courte haleine; si la réalité ne nous ennuie pas, elle nous désamorce. C'est pourquoi le coeur de nos sociétés de droits bat dans l'imaginaire, plus précisément dans la peur imaginée, qui nous transporte vers des temps et des espaces qui sont en dehors

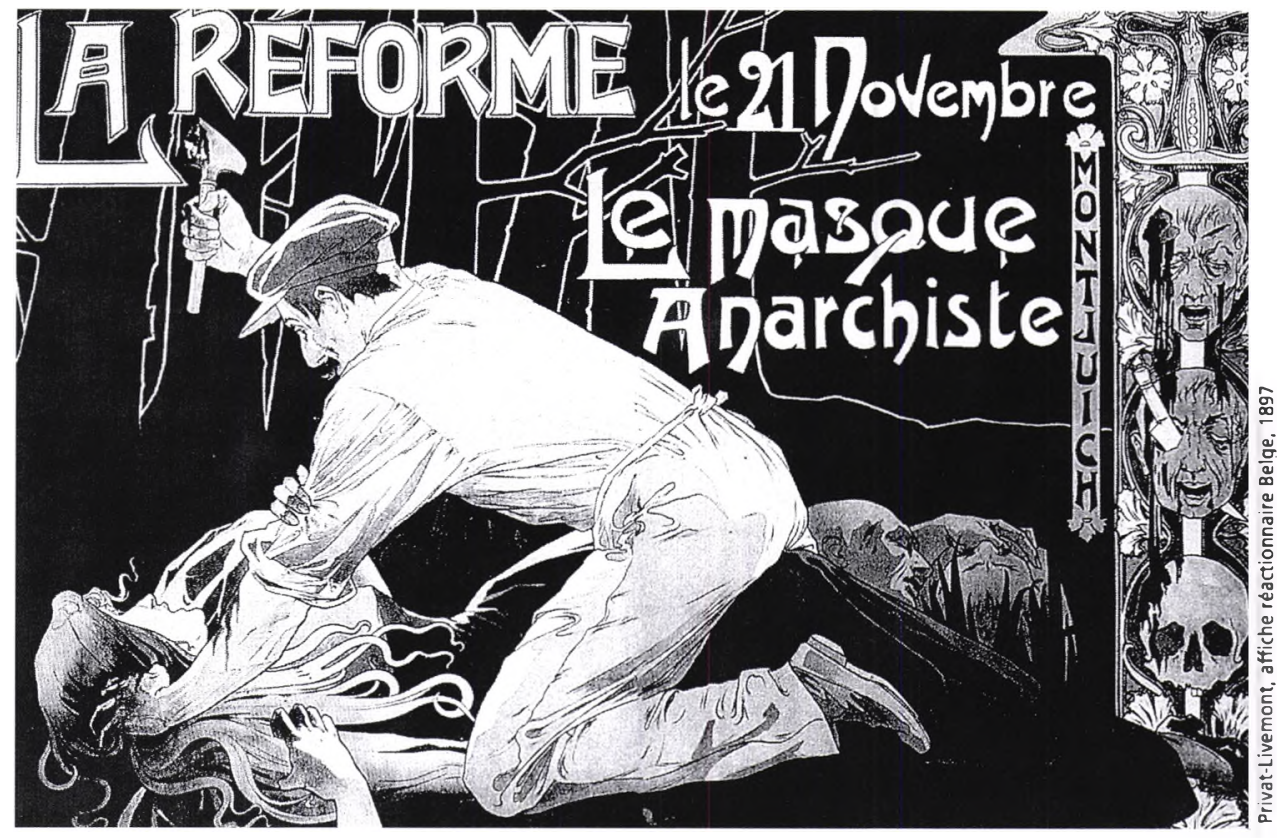

de nos vies, mais qu'il nous importe de connaître. Sans l'imaginaire, nos sociétés ne sauraient se faire des usagers du souffle qui inspira nos droits.

Dans le troisième acte de King Lear, le Duc de Cornwall arrache les yeux du Comte de Gloucester dans une scène dénuée de toute pudeur. Dans une remarquable analyse, le critique canadien Northrop Frye ${ }^{28}$ a recours à cette scène tirée de Shakespeare pour illustrer comment l'imaginaire est nécessairement lié à la vitalité de nos droits et de nos libertés:

On se souvient de la terrible scène de la pièce Le roi Lear, dans laquelle le Comte de Gloucester se fait crever les yeux sur scène. C'est une partie d'une pièce de théâtre et le théâtre est supposé être un divertissement. Maintenant, dans quel sens une scène de la sorte peut-elle être divertissante? Le fait que cela n'arrive pas en réalité est certes important. Ce serait dégradant de regarder une vraie scène d'énucléation, et encore plus d'y prendre plaisir. Par conséquent, le divertissement ne consiste pas à reproduire une authentique scène où quelqu'un se fait crever les yeux. Dans une scène théâtrale de cruauté et de haine, nous constatons la cruauté et la haine, que nous savons être un lot permanent de la vie humaine, du point de vue de l'imagination. Ce que l'imagination propose est l'horreur: non pas l'horreur paralysante d'une réelle scène d'énucléation, mais une horreur exubérante, qui est le comble de l'énergie du refus. C'est ainsi qu'en nous remémorant l'horreur devant ce qui pourrait être, l'art théâtral nous galvanise dans notre refus de perpétuer une telle horreur. (Traduction libre de la rédaction)
Le legs de la peur, en ce qu'il vit dans l'imaginaire, nous place donc devant la verticalité du bien et du mal, allant de l'abîme de la cruauté jusqu'au sommet de la rédemption. C'est cette dimension verticale de l'imaginaire qui importe; la dimension horizontale peut nous refléter notre vie avec fidélité, mais souvent sans outrage, sans susciter quoi que ce soit sinon l'indifférence. Seule la dimension verticale sait nous faire tressaillir. À ce sujet, Goethe faisait remarquer que "de ce qui fait tressaillir surgit le meilleur de l'homme». Il y a donc dans la fécondité de l'imaginaire, dans l'étude désintéressée des choses, un aliment essentiel pour l'exercice journalier du droit devant l'horreur qui peut surgir de nous. À chaque fois que nous tressaillons de peur, nous découvrons que l'émotion est toujours vraie. Rien qui dérange à ce point n'est une erreur. C'est en cela que nos démocraties dépendent ultimement de notre biologie: c'est elle et elle seulement qui nous fait tressaillir.

Notre tâche la plus impérieuse pour chacun de nous comme pour nos sociétés - consistera donc à distinguer les peurs qui nous menacent des peurs qui nous protègent. Le salut de nos libertés, de tout ce qui tend en nous vers le meilleur en dépend. Du côté des peurs qui menacent, il y a celle de Caïn qui demeura hanté par l'oeil de sa conscience jusqu'à son entrée dans la mort:

Je veux habiter sous la terre

Rien ne me verra plus, je ne verrai plus rien.

On fit donc une fosse, et Caïn

dit: C'est bien!

Puis il descendit seul sous cette voûte sombre. 
Quand il se fut assis sur sa chaise dans l'ombre

Et qu'on eut sur son front fermé

le souterrain

L'oeil était dans la tombe et

regardait Caïn ${ }^{29}$.

Puis il y a les peurs qui nous protègent. Chaque jour, nous croisons des passants anonymes qui dialoguent avec euxmêmes, cherchant ainsi à se rassurer devant une quelconque menace personnelle et qui, quand ils y parviennent abordent leur vie avec plus de confiance et de maîtrise. La littérature nous livre à l'occasion quelques échos de ces confidences. Montaigne, qui de son vivant vit se succéder trois rois de France, tous trois assassinés, et qui lui avaient accordé protection, écrivit au sujet de ces rois: "Au plus eslevé throsne du monde, nous ne sommes assis que sus notre cul ${ }^{30}$ ». Et la Sagouine nous déclame ceci au sujet du Dieu qu'elle vénère et qu'elle craint tout à la fois:

Quand c'est que je me souviens pas de ce qui vient après: «Priez pour nous pêcheurs et douneznous aujourd'hui notre pain quotidien " [...] je dis ce qui me passe par la tête et j'asseye de le dire dans des mots que le Bon Djeu peut comprendre. Ben le pus souvent, je dis rien entoute par rapport que mes genoux de laveuse qui pouvont pas endurer de rester agenouillés ben longtemps. Je finis d'accoutume par dire au Bon Djeu de pas trop se fier à moi ben de me douner la grâce de me fier à lui, ainsi-soit-il. C'est pas une prière qui se trouve dans les livres saints et j'entreprendrais pas de la dire à l'église; ben chus nous, à genoux à côté du poêle, je me figure qu'entre nous deux, le Bon Djeu est peut-être moins infinitivement fussy ${ }^{31}$.

Puis il nous reste la peur incomprise, la peur insoumise qui hante tous les étages de l'être qu'elle occupe jusqu'à l'usure du désespoir, de la vie exsangue, voire du suicide. Cette «folle en la demeure", qui orchestre au sein de notre humanité tant de drames, sait se défiler devant nos explications. Nous pouvons seulement présumer qu'elle habite là où habitent parfois les rêves, protégée de l'agression des sens par d'obliques circonvolutions cérébrales ${ }^{32}$. Parfois, quand elle relâche quelque peu son emprise, l'incomprise laisse parler ceux qu'elle habite, comme pour nous rappeler combien la souffrance qu'elle inflige est ensevelie sous un silence opaque:
Quel monstre ténébreux, étranger en moi-même,

Habite, impénétrable, à l'ombre

de mes nuits;

Au fleuve le plus vil de mon être

m'enchaîne;

M'excite quand je pars, me

retient quand je fuis;

Quand rien ne m'y contraint

dans sa course m'entraîne;

Inexorablement de flammes me

poursuit;

Dévore dans mes traits le visage

que j'aime,

Et livre à mon regard cet autre que je suis ${ }^{33}$ ?

\section{Notes}

1 Victor HUGO, La légende des siècles, Paris, Garnier-Flammarion, 1979, p. 8788 .

2 Joseph BRONOWSKI, The Ascent of Man, Boston et Toronto, Little, Brown and Company, 1973, p. 59-77.

3 Lewis MUMFORD, The City in History, New York, Harvest/HBJ. 1961, p. 26.

4 Etsuro MORI et AL., "Amygdalar Volume and Emotional Memory in Alzheimer's Disease", American Journal of Psychiatry, fév. 1999, vol. 156, no 3, p. 216-222.

5 Alexander J. MACDONALD et AL. "Cortical Afferents to the Extended Amygdala", Annals of the New York Academy of Sciences, 1999, vol. 877, p. 309-338.

6 Thackery S. GRAY, "Functional and Anatomical Relationships among the Amygdala, Basal Forebrain, Ventral Striatum, and Cortex", Annals of the New York Academy of Sciences, 1999, vol. 877, p. 439-441

7 Joseph LEDOUX, "Emotions as Memory», dans The Handbook of Emotion and Memory, sous la direction de S.A. CHRISTIANSEN, Hillsdale N.J., L. Erhart Publisher, p. 269-288.

8 Sigmund FREUD, «Introductory Lecture on Psycho-Analysis» (1916-1917) dans The Standard Edition of the Complete Psychological Works of Sigmund Freud, sous la direction de J. STRACHEY, London, Hogart Press, 1961.

9 William B. SCOVILLE et Brenda MILNER, "Loss of Recent Memory after Bilateral Hippocampal Lesions", Journal Neurol. Neurosurg. Psychiatry, 1957, vol. 201, p. 11-21.

Brenda MILNER, Larry R. SQUIRE, Eric KANDEL, "Cognitive Neuroscience and the Study of Memory", Neurol. Behavior, 1998, vol. 20, p. 445-468

10 Larry R. SQUIRE, "The Medial Temporal Lobe in Memory", Science, 1996, vol. 253. p. $1380-1386$

11 Sigmund FREUD, "Inhibition, Symptoms and Anxiety" (1926) dans The Standard Edition of the Complete Psychological Works of Sigmund Freud, sous la direction de J. STRACHEY, London, Hogart Press, 1961.

12 Ivan P. PAVLOV, Experimental Psycho logy and Other Essays, New York, Philosophical Library, 1957.

13 Barry J. EVERETT et AL., "Associative Process in Addiction and Reward: The Role of the Amygdala-Ventral Striatal Subsystem", Advancing from the Ventral Striatum to the Extended Amygdala, sous la direction de Jaqueline MCGINTY, Annals of the New York Academy of Sciences, 1999, vol. 877, p. 412-438.
14 Leo KAMIN, "Predictability, Surprise, Attention, and Conditioning", dans, Punishment and Aversive Behavior, sous la direction de B. A. CAMPBELL et R. M. CHURCH, 1969, New York, Appleton Century Crafts, p. 279-296.

15 René SPITZ, "Hospitalism: An Inquiry into the Genesis of Psychiatric Conditions in Early Childhood", dans, Psychoanalytic Study of the Child, 1945, vol. 1, p. 53-74

16 John BOWLBY, Attachment and Loss, vol. 1, Attachement, Part V, Chapter 19, New York, Basic Books, (1969) 1982, 2e édition rev. et augm., p. 372-374.

17 Hans SELYE, "A Syndrome Produced by Diverse Nocious Agents", Nature, 1936, vol. 138, p. 22-36.

18 Samuel LEVINE et AL., "Physiological and Behavioral Effects of Infantile Stimulation", Physiol. Behav., 1967, vol. 2, p. 55-63.

19 Bruce MCEWEN et Robert SAPOLSKY "Stress and Cognitive Function", Current Opinion in Neurobiology, 1995, vol. 5, p. 205-216.

20 Alix STRACHEY, The Unconscious Motives of War, 1957, New York, International University Press, p. 424.

21 Lewis MUMFORD, ibid. note 3, p. 106 108.

22 John BOARDMAN et AL., The Roman World, New York, Londres, Oxford University Press, 1988, p. 34-35.

23 John LOCKE, The Second Treatise on Civil Government, London, Prometheus Books, 1986, p. 34

24 Thomas HOBBES, Leviathan, Cambridge Cambridge University Press, 1996, p. 85 .

25 Gennadi GERASIMOV, "A Death Wish is Haunting Russia", New York Times, 16 décembre 1994, p. A22.

26 Eugène BONNEMẼRE, Histoire des Paysans, Paris, Sandoz et Fishbacher, 1874 , p. 41.

27 Déclaration universelle des droits de l'homme, Nations Unies, New York.

28 Northrop FRYE, The Educated Imagination, Bloomington, Indiana University Press, 1964, p. 98-99

29 Victor HUGO, ibid. note 1, p. 88

30 Michel DE MONTAIGNE, Essais, Paris, Gallimard, 1965

31 Antonine MAILLET, La Sagouine, Ottawa, Leméac, 1971 , p. 69.

32 John A. HOBSON et AL., "To Dream or Not to Dream? Relevant Data from New Neuroimaging and Electrophysiological Studies", Current Opinion in Neurobiolgy, 1998, avril, vol. 8, p. 239-244.

33 Maurice COURANT, Ténébreuse lumière, Paris, Éditions de la Revue Moderne, 1979, p. 13.

\section{Suggestion de lecture}

Richard BROCKMAN, A Map of the Mind: Toward a Science of Psychotherapy, Madison, Connecticut, Psychosocial Press, 1999

Ce livre a le mérite de présenter une synthèse souvent tentée sans succès: le lien entre les apports des sciences neurologiques et la dynamique psychothérapeutique. L'auteur arrive à faire cette synthèse et offre une élégante analyse des soubresauts de la psychothérapie à partir d'une perspective neurobiologique, tout particulièrement au sujet de l'impact de la peur.

L'auteur veut remercier Hélène Laberge, Jacques Dufresne, Pierre Migneault, et Jean-Claude Leblond, Diane Laflamme, et Luce Des Aulniers pour leurs lec tures critiques et sympathiques de ce texte. 